\title{
Type I and II Diabetic Adipose-Derived Stem Cells Respond In Vitro to Dehydrated Human Amnion/ Chorion Membrane Allograft Treatment by Increasing Proliferation, Migration, and Altering Cytokine Secretion
}

\author{
Michelle Massee, ${ }^{*}$ Kathryn Chinn, ${ }^{*}$ Jeremy J. Lim, Lisa Godwin, \\ Conan S. Young, and Thomas J. Koob \\ MiMedx Group, Inc., Marietta, Georgia. \\ *These authors contributed equally to this work.
}

Objective: Human amniotic membranes have been shown to be effective for healing diabetic foot ulcers clinically and to regulate stem cell activity in vitro and in vivo; however, diabetic stem cells may be impaired as a sequela of the disease. In this study, dehydrated human amnion/chorion membrane (dHACM) allografts (EpiFix ${ }^{\circledR}$; MiMedx Group) were evaluated for their ability to regulate diabetic stem cells in vitro.

Approach: Human adipose-derived stem cells (ADSCs) from normal, type I diabetic, and type II diabetic donors were treated with soluble extracts of dHACM and evaluated for proliferation after 3 days by DNA assay, chemotactic migration after 1 day by transwell assay, cytokine secretion after 3 days by multiplex ELISA, and gene expression after 5 days by reverse transcription-polymerase chain reaction.

Results: Although diabetic ADSCs demonstrated decreased responses compared to normal ADSCs, AHACM treatment stimulated diabetic ADSCs to proliferate after 3 days and enhanced migration over $24 \mathrm{~h}$, similar to normal ADSCs. dHACM-treated diabetic ADSCs modulated secretion of soluble signals, including regulators of inflammation, angiogenesis, and healing. All ADSCs evaluated also responded to dHACM treatment with altered expression of immunomodulatory genes, including interleukins (IL)-1 $\alpha$, IL-1 $\beta$, and IL-1RA.

Innovation: This is the first reported case demonstrating that diabetic ADSCs respond to novel amniotic membrane therapies, specifically treatment with dHACM. Conclusion: $\mathrm{dHACM}$ stimulated diabetic ADSCs to migrate, proliferate, and alter cytokine expression suggesting that, despite their diabetic origin, ADSCs may respond to dHACM to accelerate diabetic wound healing.

\section{INTRODUCTION}

DiabeTES MELlitus (DiABETES) is a chronic disease affecting 347 million people globally and causes complications that lead to both patient mor- bidity and mortality. Type I diabetes results from the patient's body failing to produce enough insulin, and type II diabetes results from the patient's cells failing to respond to in-

(c) Michelle Massee, et al. 2015; Published by Mary Ann Liebert, Inc. This Open Access article is distributed under the terms of the Creative Commons Attribution Noncommercial License (http:// creativecommons.org/licenses/by-nc/4.0/) which permits any noncommercial use, distribution, and reproduction in any medium, provided the original author(s) and the source are credited.

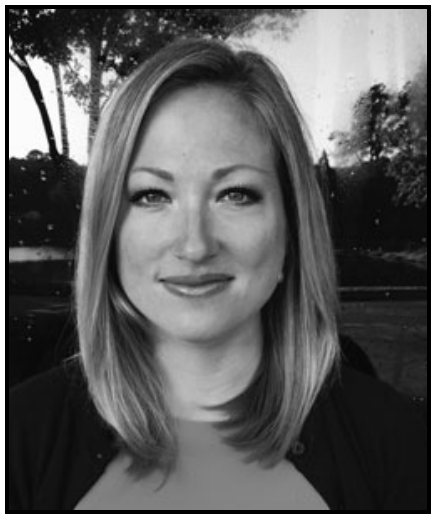

Michelle Massee, BS

Submitted for publication May 11, 2015. Accepted in revised form June 29, 2015.

*Correspondence: MiMedx Group, Inc., 1775 West Oak Commons Court NE, Marietta, GA 30062 (e-mail: mmassee@mimedx.com) 
sulin properly. ${ }^{1} \mathrm{Up}$ to $25 \%$ of all people with diabetes will develop chronic wounds, which without proper treatment are the cause of $70-84 \%$ of lower limb amputations. ${ }^{2}$

The process of wound repair is a complex sequence where molecular signaling and cellular events facilitate the transitions into and out of each phase of wound repair. Diabetes impairs both extrinsic and intrinsic factors, including availability of important protein factors and cellular responsiveness, both proximally and distally to the wound that disrupts healing. ${ }^{3}$ Repeated trauma applied to an extremity that has been rendered insensitive, ${ }^{4}$ combined with reduced bioavailability and abnormal expression of cytokines and growth factors, has been implicated in the development of a chronic inflammatory state, ${ }^{5,6}$ thus resulting in a wound that is unable to progress toward healing.

Scientific literature suggests that bone marrowderived mesenchymal stem cells (BM-MSCs) may facilitate wound healing and modulate inflammation. ${ }^{7}$ Since adipose-derived stem cells (ADSCs) are closely related to BM-MSCs and more easily obtained, 8,9 ADSCs are being explored as a therapeutic option for accelerating wound repair and regeneration. Multiple in vitro and in vivo studies demonstrate that ADSCs mediate several aspects of the healing cascade through the paracrine secretion of growth factors and cytokines. ${ }^{10-12}$ The unique secretome of ADSCs possesses immunomodulatory capabilities, which can partly be attributed to the production of hepatocyte growth factor (HGF), granulocyte and macrophage colony stimulating factors (G-CSF, GM-CSF), interleukins (IL-) 6, 7, 8, and 11 , tumor necrosis factor- $\alpha$ (TNF- $\alpha)$ BDNF, adipokines, along with others. ${ }^{9,13}$ In addition, studies have identified ADSCs' role in tissue regeneration through their secretion of vascular endothelial growth factor (VEGF), HGF, transforming growth factor-beta (TGF- $\beta$ ), and stromal cell-derived factor $1 \alpha(\mathrm{SDF}-1 \alpha) .{ }^{14}$ Successful revascularization of the wound is crucial for transitioning out of the inflammation phase by providing the granulation bed for tissue formation and remodeling. These studies suggest that ADSCs may be a key cell type for soft tissue regeneration because they are capable of modulating several aspects of healing. However, limited studies have been performed to characterize the effect of diabetes on stem cells. A handful of studies indicated a decrease in ADSC population doublings when cultured in type II diabetic conditions ${ }^{15}$ and decreased proliferative and migratory potential in ADSCs derived from streptozotocin-induced type I diabetic mice. ${ }^{16}$ ADSCs from diabetic mice also released lower amounts of HGF, VEGF-A, and insulin-like growth factor-1 (IGF-1), all of which play important roles in wound repair and regeneration. ${ }^{16}$ These studies suggest that diabetes alters ADSCs' biological properties and impairs their function in wound healing.

Previous studies demonstrated that the dehydrated human amnion/chorion membrane (dHACM), comprising of gently cleansed laminated amnion and chorion membranes, was able to stimulate cellular proliferation and migration responses in a variety of adult stem cells from normal donors, including BM-MSCs, ADSCs, and hematopoietic stem cells, ${ }^{17}$ suggesting that dHACM contains soluble components that elute from the tissue and stimulate the growth and chemotactic migration of different types of stem cells. ${ }^{18-20}$ Following these observations and reported clinical outcomes demonstrating that diabetic foot ulcers (DFUs) respond positively to treatment with dHACM, this study was undertaken to evaluate whether similar cellular responses could be observed in type I and II diabetic ADSCs in combination with dHACM to better understand the mechanisms by which dHACM facilitates healing in diabetic tissues. Human ADSCs harvested from type I and II diabetic donors were characterized and assessed for proliferation, migration, cytokine expression, and secretion in response to treatment with soluble dHACM extracts. The hypothesis being tested was that $\mathrm{dHACM}$ can rescue the impaired function of ADSCs in diabetics to advance normal healing of chronic wounds.

\section{CLINICAL PROBLEM ADDRESSED}

Diabetes is a chronic disorder affecting an ever increasing population of people. A confounding result of this disease is the manifestation of chronic wounds that occur in $25 \%$ of people afflicted with diabetes. ${ }^{2}$ Standard protocol for treating chronic wounds, as defined by a wound failing to heal within 4 weeks, results in $\sim 8 \%$ of ulcers healing in a 12 -week period. ${ }^{21}$ Of the wounds that do not heal, a significant portion leads to chronic infection and ultimately limb amputation. Therefore, advanced wound therapies are in use to combat the complications associated with diabetes.

\section{MATERIALS AND METHODS}

Proliferation, migration, growth factor secretion, and gene expression following exposure to dHACM extract were tested in ADSCs derived from type I 
and type II diabetic donors in comparison to ADSCs derived from a healthy normal donor.

Normal ADSCs (Female, 46Y, BMI 22, PT5006; Lonza), type I diabetic ADSCs (Female, 62Y, BMI 22, PT5007; Lonza), and type II diabetic ADSCs (Female, 72Y, BMI 29, PT5008; Lonza) were cultured in complete media, prepared by adding the ADSC supplement kit (final concentrations: $10 \% \mathrm{v} / \mathrm{v}$ fetal calf serum, $1 \% \mathrm{v} / \mathrm{v}$ L-glutamine, $30 \mu \mathrm{g} / \mathrm{mL}$ gentamicin, $15 \mathrm{ng} / \mathrm{mL}$ amphotericin B, PT4503; Lonza) to the ADSC medium (PT3273; Lonza). Basal media used in cell treatments were prepared by adding only the gentamicin/amphotericin and L-glutamine components of the ADSC supplement kit to ADSC media and excluding the serum supplement.

\section{Processing of dHACM}

dHACM (EpiFix ${ }^{\circledR}$; MiMedx Group, Inc., Marietta, GA) is a dehydrated human tissue allograft comprising laminated amnion and chorion membranes derived from the human placenta. Human placentas were donated under informed consent following caesarean sections, as regulated by the Food and Drug Administration's (FDA) Good Tissue Practice and American Association of Tissue Banks (AATB). Amnion and chorion tissue layers were isolated from the placenta, processed using a proprietary process that involves gentle cleansing of the layers, and then laminated to form the graft, which was dehydrated and sterilized under controlled conditions. ${ }^{22}$

\section{Preparation of dHACM extracts}

To prepare soluble extracts of dHACM for cell culture experiments, dHACM grafts were minced yielding $\sim 1 \times 1 \mathrm{~mm}$ pieces of tissue, and soluble factors from the membranes were allowed to elute into ADSC basal media at an appropriate concentration. After overnight extraction at $4^{\circ} \mathrm{C}$, the tissue residue was removed by centrifugation at 3,000 RCF for 5 min at room temperature, and the extract was sterile filtered using a $0.22-\mu \mathrm{m}$ filter unit (SLMP025SS; EMD Millipore). Previous studies have established that growth factors and cytokines in dHACM elute from the tissue under these conditions. ${ }^{18}$ It is this fraction that is the initial biological component being delivered with the application of the graft; therefore, this model examines the response of normal and diabetic ADSCs to the numerous array of soluble factors contained within dHACM.

\section{ADSC proliferation}

Normal, type I, and type II ADSCs were plated separately at 2,500 cells/well in 96-well plates in complete media and were allowed to adhere to tissue culture-treated plastic overnight. The media were then removed and the wells were washed with sterile phosphate buffered saline (PBS, SH30028.01; GE Healthcare) to remove unattached cells and serum components. Both healthy and diseased cell types were treated with $\mathrm{dHACM}$ extract at 10,5 , and $1 \mathrm{mg}$ tissue per $\mathrm{mL}$ of basal media, which were prepared by making serial dilutions of the original $10 \mathrm{mg} / \mathrm{mL}$ extract in basal media $(n=5)$. The positive and negative control wells were treated with complete media containing serum supplements and unsupplemented basal media, respectively. After $72 \mathrm{~h}$, the treatment media were collected and frozen at $-80^{\circ} \mathrm{C}$ for growth factor secretion analysis from each cell type. The plate was washed with PBS to remove unattached cells and residual media. A CyQUANT assay (C7026; Molecular Probes, Life Technologies Corp.) was performed according to the manufacturer's instructions, to quantify DNA content ( $n=5$ replicates of samples per treatment) as a measure of cellular proliferation. DNA content was translated to cell number, using a standard curve of known quantities of cells as determined by counting on a hemocytometer and measuring with the $\mathrm{Cy}$ QUANT assay.

\section{ADSC migration}

Treatment groups, including dHACM extract, serum-containing positive controls, and serum-free negative controls, were prepared in the bottom of 24-well plates embedded in agarose gels by mixing $100 \mu \mathrm{L}$ of treatment and $100 \mu \mathrm{L}$ of $1 \%$ agarose, yielding a final concentration of $0.5 \%$ agarose containing dHACM extract, serum containing medium, or basal medium. Treatment groups of dHACM were prepared at 10,5 , and $2 \mathrm{mg} / \mathrm{mL}$ as a final concentration of the total media volume by dilution of $80 \mathrm{mg} / \mathrm{mL}$ dHACM extract with basal media. The positive controls were also prepared in agarose gels with newborn calf serum (NCS) at a $10 \%$ final concentration of the total medium volume. The negative controls were prepared by combining $100 \mu \mathrm{L}$ basal media with $100 \mu \mathrm{L}$ of $1 \%$ agarose gel. The agarose was allowed to gel at $4^{\circ} \mathrm{C}$ for $45 \mathrm{~min}$ to immobilize the treatments to the bottom of the wells in plates wrapped in Parafilm (Bemis Company, Inc.) to prevent drying. Four hundred microliters of basal ADSC media was added to each of the gelled treatment wells to permit a diffusion of soluble factors out of the gels and into the media.

Normal, type I, and type II ADSCs were serum starved in basal media for $24 \mathrm{~h}$ before plating and were treated with $10 \mu \mathrm{g} / \mathrm{mL}$ mitomycin C (MMC, AB120797; Abcam) for $2 \mathrm{~h}$ before plating to inhibit DNA synthesis and cellular division. Preliminary 


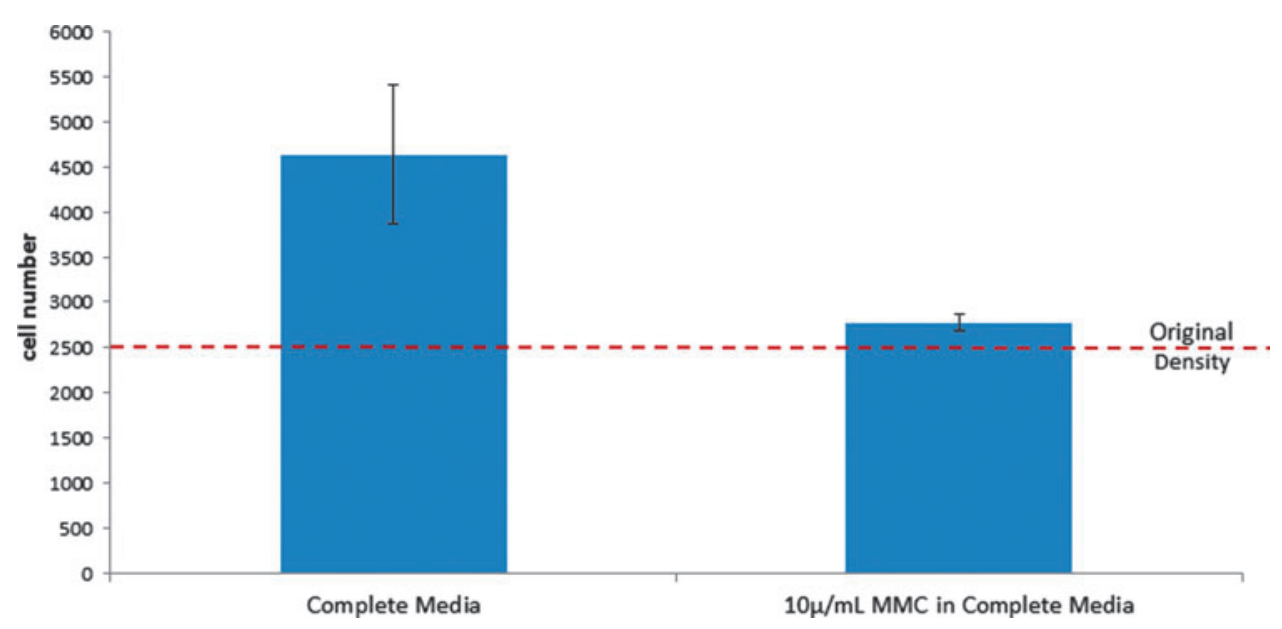

Figure 1. Response of normal ADSCs to MMC treatment after $24 \mathrm{~h}$. Each bar represents the average number of cells per treatment $(n=5)$. The dashed line indicates the original seeding density. Error bars indicate standard deviation from mean values. ADSCs, adipose-derived stem cells; MMC, mitomycin C.

studies validated that the MMC treatment parameters fully arrested cellular division (Fig. 1), following pretreatment of the cells with MMC and quantifying the cell numbers after $24 \mathrm{~h}$. Cell proliferation was suppressed following $10 \mu \mathrm{g} / \mathrm{mL}$ MMC treatment.

Each cell type was plated at 40,000 cells per $8.0-$ $\mu \mathrm{m}$-pore size transwell insert (07-200-150; Corning) in $200 \mu \mathrm{L}$ basal media $(n=4)$. The transwell inserts containing cell suspensions were then aseptically transferred into the treatment wells. The cells were allowed to migrate for $24 \mathrm{~h}$ at $37^{\circ} \mathrm{C}$.

After $24 \mathrm{~h}$ in culture, the media were removed from the tops of each transwell insert. The inside of each insert was swabbed gently with a cotton-tipped swab and rinsed with Hank's Balanced Salt Solution (HBSS) with $\mathrm{Ca}^{2+}$ and $\mathrm{Mg}^{2+}$ (SH3026801; GE Healthcare). Each insert was placed into $500 \mu \mathrm{L}$ of HBSS to rinse any residual media from the bottom of the insert membranes. The inserts were incubated in $500 \mu \mathrm{L}$ of TrypLE dissociation solution (12563; Gibco) at $37^{\circ} \mathrm{C}$ for $30 \mathrm{~min}$ to remove migrated cells from the bottom of the insert membranes. Preliminary experiments confirmed that this treatment removed all cells from the membranes.

The CyQUANT assay was used by diluting the dye $1: 75$ in $4 \times$ lysis buffer to determine relative DNA quantities of ADSCs, which migrated to the bottom surface of the transwell inserts. Inserts were also stained with DAPI as a visual confirmation of the migration response.

\section{Stem cell cytokine, chemokine, and growth factor modulation}

Protein secretion from normal, type I diabetic, and type II diabetic ADSCs was quantified following $72 \mathrm{~h}$ of dHACM extract treatment at 10,5 , and

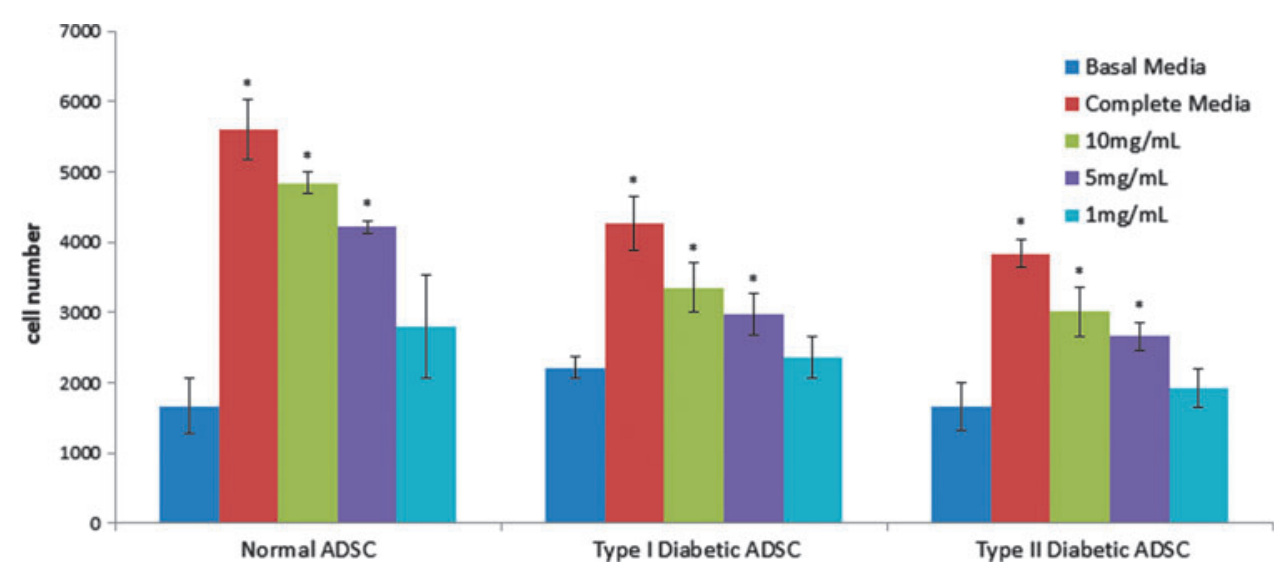

Figure 2. Proliferation of normal, type I diabetic, and type II diabetic ADSCs over $72 \mathrm{~h}$ in response to treatment with soluble extracts of dHACM, as well as negative and positive controls. Each bar represents the average number of cells per treatment for each cell type $(n=5)$. Error bars indicate standard deviation from mean values. ${ }^{*} p \leq 0.05$, where indicated versus basal media control. dHACM, dehydrated human amnion/chorion membrane. 
$1 \mathrm{mg} / \mathrm{mL}$ in comparison to complete and basal medium treatments. Cell numbers were measured in each well using a CyQUANT assay after the culture media were collected, and treatment media were analyzed for cytokine content using Quantibody ${ }^{\circledR}$ Human Growth Factor and Inflammation Arrays (QAH-CAA-1000; RayBiotech). The amount of each growth factor in the original extracts was measured and subtracted from that in the media to calculate the amount of additional growth factor produced by the cells themselves in response to dHACM extract treatment. Growth factor production values were then normalized on a per cell number basis to account for differential effects on proliferation from the different conditions.

\section{Gene expression of diabetic ADSCs}

Gene expression of normal and diabetic ADSCs was compared by reverse transcription-polymerase chain reaction. Normal, type I diabetic, and type II diabetic ADSCs (passage 3) were cultured in complete media in T-25 culture flasks. RNA was then extracted using a GeneJET RNA Purification Kit (Thermo Scientific) and quantified using a NanoDrop 2000 spectrophotometer (Thermo Scientific). Reverse transcription to cDNA was performed using a Maxima First Strand cDNA Synthesis Kit (Thermo Scientific).

Quantitative polymerase chain reaction amplification for each gene target was performed on a QuantStudio 7 System (Life Technologies) using TaqMan Gene Expression Arrays for Human Diabetes, Human Growth Factors, and Human Extracellular Matrix (ECM) and Adhesion Molecules (Life Technologies). When combined, these arrays contain validated primers and probes for 228 different gene targets related to diabetes, growth factors, ECM, and adhesion molecules. The Human Diabetes Array assayed gene expression for 44 genes related to insulin, insulin resistance, obesity, and maintenance of blood sugar levels, as well as transcription factors, signal transduction proteins, transporters, and receptors associated with diabetes. The Human Growth Factors Array compared 92 growth factor proteins involved in cell differentiation, regulation of apoptosis, angiogenesis, embryonic development, and tissuespecific development. The Human ECM and Adhesion Molecules Array examined 92 genes involved in ECM structure and remodeling, defining connective tissue, cell adhesion, transmembrane inhibitors, basement membrane construction, and collagen proteins. To determine relative expression of diabetic cells over normal ADSCs, the $2^{-\Delta \Delta \mathrm{Ct}}$ comparative $\mathrm{Ct}$ method of relative quantification ${ }^{23}$ was used with eukaryotic 18S ribosomal RNA, glyceraldehyde-3- phosphate dehydrogenase (GAPDH), glucuronidase beta (GUSB), and hypoxanthine phosphoribosyltransferase 1 (HPRT1) as endogenous control genes.

\section{Gene expression of dHACM-treated ADSCs}

Normal, type I diabetic, and type II diabetic ADSCs (passage 3) were plated in 24-well plates at 2,500 cells $/ \mathrm{cm}^{2}$ in complete medium $(n=4)$. After $24 \mathrm{~h}$ to allow for cell adhesion, ADSCs were rinsed with PBS and treated with dHACM extracts at 10, 5 , and $1 \mathrm{mg} / \mathrm{mL}$ for 5 days. Complete and basal media were used as positive and negative controls, respectively. Following 5 days of treatment, RNA from ADSCs was extracted, quantified, and reverse transcribed as described previously. Gene expression was determined using predesigned TaqMan assays (Life Technologies) for $I L-1 \alpha$ (Assay ID: Hs00174092_m1), IL-1 $\beta$ (Assay ID: Hs01555410_ $\mathrm{m} 1)$, IL-1 receptor antagonist (IL-1RA, Assay ID: Hs00893626_m1), TNF- $\alpha$ (Assay ID: Hs00174128_ $\mathrm{m} 1$ ), prostaglandin E synthase (PTGES, Assay ID: Hs01115610_m1), TGF- $\beta 1$ (Assay ID: Hs00998133_ $\mathrm{m} 1$ ), and HGF (Assay ID: Hs00300159_m1). The $2^{-\Delta \Delta \mathrm{Ct}}$ method $^{23}$ was used to determine relative expression of dHACM-treated ADSCs over ADSCs in the basal medium with eukaryotic $18 \mathrm{~S}$ ribosomal RNA (18S, Assay ID: Hs99999901_s1) as an endogenous housekeeping control.

\section{Statistical analyses}

All values are reported as mean \pm standard deviation. For statistical analyses, analysis of variance was used to determine statistical significance of groups, and Tukey's post hoc multiple comparison test with significance set at $p \leq 0.05$ indicated significance between individual samples. Statistical analyses were carried out using Minitab (v17.1).

\section{RESULTS dHACM stimulates proliferation of diabetic ADSCs}

The normal, type I diabetic, and type II diabetic ADSCs were tested for their ability to respond to soluble factors in dHACM extracts in an in vitro cellular proliferation assay. All cell types tested were found to proliferate at low levels after $72 \mathrm{~h}$ of culture in basal media, whereas inclusion of calf serum in the positive control wells caused the cells to at least double in number for all cell types compared to basal media controls (Fig. 2). The comparison of the complete media controls for each cell type demonstrated that the diabetic cells had slower proliferation rates over $72 \mathrm{~h}$ compared to normal ADSCs. The normal ADSCs in the calf 


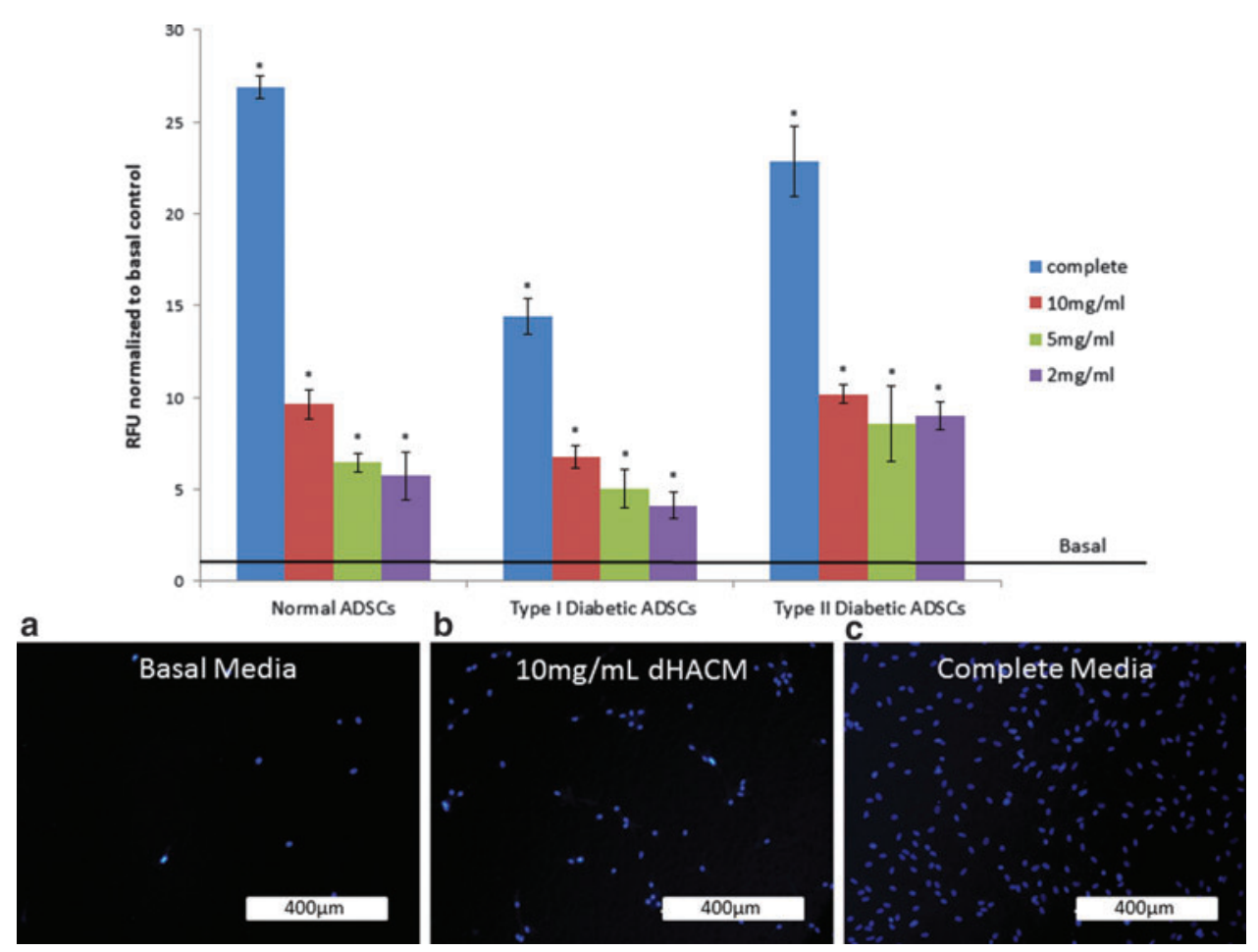

Figure 3. Migration of MMC pretreated normal, type I diabetic, and type II diabetic ADSCs over $24 \mathrm{~h}$ in response to treatment with soluble extracts of dHACM extracts embedded in a $0.5 \%$ agarose gel, as well as basal medium and complete medium controls. The negative control wells contained basal medium, while the positive control wells were supplemented with newborn calf serum. Each bar represents the relative fluorescent units (RFUs) representing the average number of cells per treatment for each cell type normalized to basal medium controls, which is represented by a black line in the graph $(n=5)$. Error bars indicate standard deviations from mean values. ${ }^{*} p \leq 0.05$, where indic ated versus negative control. Separate wells were designated for DAPI staining as a visual confirmation of the migration response. (a) Representative image of migration of normal ADSCs in response to basal medium in agarose. (b) Representative image of migration of normal ADSCs in response to $10 \mathrm{mg} / \mathrm{mL}$ dHACM extract in agarose. (c) Representative image of migration in response to serum in agarose.

serum proliferated to an approximately fourfold greater extent compared to their basal medium controls, whereas type I and type II diabetic cells in identical conditions resulted in an approximate twofold increase in cell number over basal controls

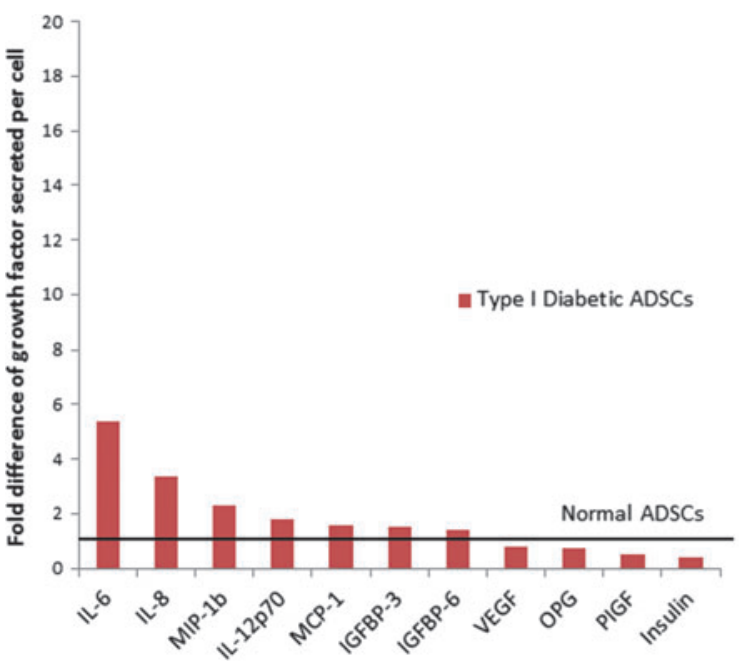

over the same time period. These data indicate that the diabetic ADSCs evaluated in this study exhibited an impaired proliferative response relative to normal ADSCs under the conditions of the assay.

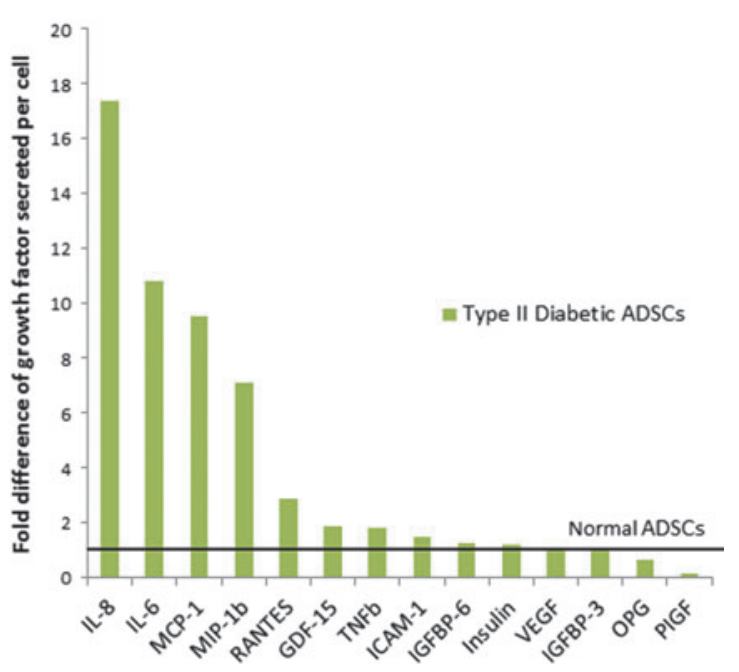

Figure 4. Growth factor secretion by type I and type II diabetic ADSCs, normalized to normal ADSCs, in complete medium. Data for type I and II diabetic ADSCs were normalized to quantities of each factor measured for normal ADSCs, the latter of which is represented by a black line in the graph. 
All dHACM extracts were prepared in basal media; therefore, any cellular proliferation over levels observed in basal media was attributed to the soluble components provided by dHACM. Incubation with dHACM extracts resulted in increased proliferation of normal, type I diabetic, and type II diabetic ADSCs in a dose-dependent manner, as seen in Fig. 2. Cell proliferation was highest after $72 \mathrm{~h}$, for all cell types, in the wells containing $10 \mathrm{mg} / \mathrm{mL}$ extract $(p \leq 0.05)$, followed by $5 \mathrm{mg} / \mathrm{mL}$ $(p \leq 0.05), 1 \mathrm{mg} / \mathrm{mL}$, and basal media only.

\section{dHACM increases migration of diabetic ADSCs}

The cellular migration results demonstrated that complete media (10\% NCS), 10,5 , and $2 \mathrm{mg} / \mathrm{mL}$ of dHACM extract, stimulated migration of normal healthy ADSCs, type I diabetic, and type II diabetic ADSCs through a porous transwell membrane after $24 \mathrm{~h}$ compared to the basal control group $(p \leq 0.05)$. The comparison of the complete media controls for each cell type demonstrated that type I diabetic cells experienced a significantly lower migration over $24 \mathrm{~h}$ compared to normal ADSCs in complete media, confirming that the type I ADSCs from this donor were impaired for their ability to migrate under the assay conditions employed. The normal ADSCs migrated to an approximately twofold greater extent compared to type I ADSCs in complete media; whereas type II ADSCs in identical conditions were similar to normal ADSCs.

Despite the type I diabetic ADSCs that impeded migration potential in comparison to cells from a healthy donor, the cells were stimulated by the chemotactic signals eluted from dHACM to increase migration through the transwell membrane in a dose-dependent manner, relative to their basal medium controls $(p \leq 0.05)$. Normal and type II diabetic ADSCs also responded in a dose-dependent manner to dHACM treatment (Fig. 3), compared to their basal controls $(p \leq 0.05)$.

\section{dHACM alters stem cell cytokine, chemokine, and growth factor secretion by diabetic ADSCs}

The regulation of normal, type I diabetic, and type II diabetic ADSCs was further investigated by measuring the in vitro growth factor and cytokine levels secreted by the cells following a 3-day treatment with dHACM extract. The conditioned media were collected from each treatment group and analyzed using the Quantibody ${ }^{\circledR}$ Multiplex ELISA platform.

The complete media treatment represented the natural growth factor secretion profile of each cell type in optimal conditions and was used to high- light the inherent differences in proteins secreted between ADSCs from healthy and diabetic donors in vitro. A number of proteins, including IL-8, IL-6, monocyte chemoattractant protein-1 (MCP-1), and macrophage inflammatory protein-1 (MIP-1 $\beta$ ), were more highly secreted by type I and II diabetic ADSCs than normal ADSCs, while osteoprotegerin (OPG) and placental growth factor (PlGF) were secreted at lower levels by diabetic cells (Fig. 4).

The protein secretion impact of dHACM on healthy and diabetic ADSCs was assessed through the identification of factors that experienced at least a 10-fold change over basal detection levels, representing at least one order of magnitude difference. The data are compiled as a heat map with upregulation represented as a green color gradient and downregulation represented as a red color gradient (Fig. 5). For all cell types, a large number of growth factors and cytokines were impacted by treatment with dHACM over the basal control treatment. Type I diabetic ADSCs were found to also upregulate immunoregulatory proteins: IL6, IL-8, Regulated on Activation Normal T Cell Expressed and Secreted (RANTES), MCP-1, GCSF, insulin-like growth factor-binding protein 1 (IGFBP-1), growth differentiation factor 15 (GDF-15), and MIP-1 $\beta$ in a dose-dependent manner to dHACM. Type II diabetic ADSCs treated with $10 \mathrm{mg} / \mathrm{mL}$ of dHACM extract were found to upregulate immunoregulatory proteins: IL-6, IL-8, IL-11, RANTES, MCP-1, IGFBP-1, GDF-15, MIP-1 $\beta$, G-CSF, stem cell factor (SCF), VEGF, TGF $\beta-1,-3$, nerve growth factor receptor (NGFR), and IGF-1. Upregulation of IL-6, -8, RANTES, MCP-1, and G-CSF was commonly observed in all ADSCs evaluated in response to dHACM extracts. Type I diabetic ADSCs downregulated SCF R, -IL-12p70, IGFBP-6, TGF $\beta 3$, IGFBP-3, insulin, eotaxin, and tissue inhibitor of metalloproteinase-1 and 2 (TIMP-1 and TIMP-2) in response to dHACM. Type II diabetic ADSCs downregulated IGFBP-6, HGF, TIMP-1, and TIMP2 in response to dHACM. Downregulation of SCF R, TIMPs 1 and 2, and IGFBP-6 was common to all ADSCs evaluated in response to dHACM extracts.

\section{dHACM regulates gene expression of immunomodulatory factors by diabetic ADSCs}

Gene expression of type I and II diabetic ADSCs in complete medium demonstrated that a wide variety of genes related to ECM and adhesion, growth factors and cytokines, and diabetes were differentially regulated in diabetic cells compared to normal ADSCs. In particular, gene targets related to immunomodulation were highly 


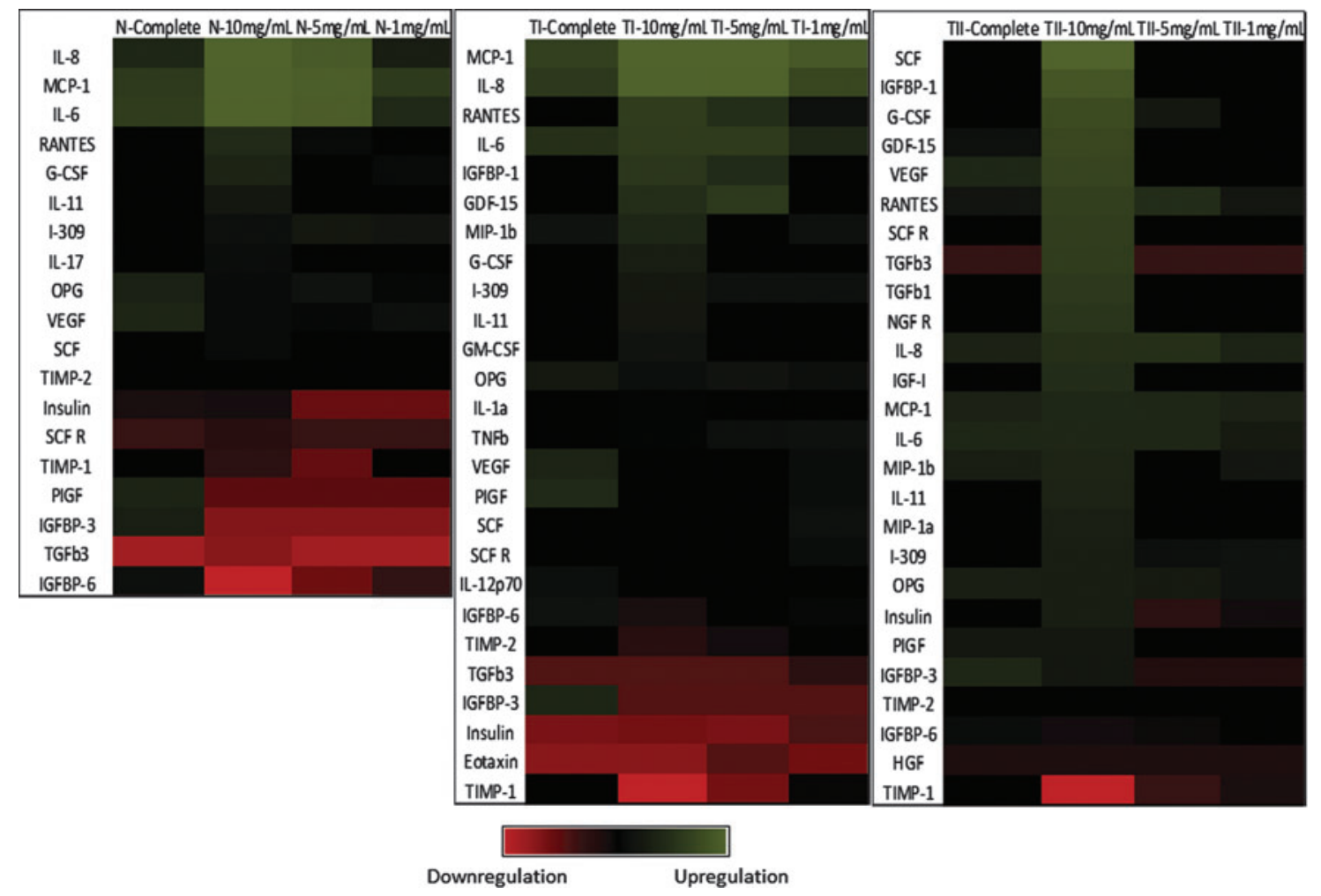

Figure 5. Cytokine secretion by dHACM-treated ADSCs. The data are presented as a heat map with upregulation relative to basal media represented as a green color gradient and downregulation represented as a red color gradient. N, normal ADSCs; TI, type 1 diabetic ADSCs; TII, type 2 diabetic ADSCs.

upregulated in diabetic cells, including $I L-1 \alpha$ (28.6-fold upregulation in type I, 55.0-fold in type II), $I L-1 \beta$ (3.8-fold in type I, 19.9-fold in type II), and $T N F-\alpha$ (5.6-fold in type I, not detected in type II). Therefore, these targets along with additional targets related to immunomodulation were further examined at the level of gene expression in normal and diabetic ADSCs following dHACM treatment.

Gene expression analysis of dHACM-treated ADSCs demonstrated that normal, type I diabetic, and type II diabetic ADSCs were responsive to treatment with dHACM extracts after 5 days. Gene expression related to immunomodulation by ADSCs was variably regulated following dHACM treatment. $I L-1 \alpha$ and $I L-1 \beta$ expression was commonly upregulated by all ADSCs following dHACM treatment in a dose-dependent manner (Fig. 6a, b). Interestingly, $I L-1 R A$, the receptor antagonist of IL-1, was also significantly upregulated in normal and diabetic ADSCs with $10 \mathrm{mg} / \mathrm{mL}$ dHACM extract treatment (Fig. 6c). For comparison, in complete medium that represented the standard culture conditions for these cells, normal and diabetic ADSCs consistently had higher expression of $I L-1 \alpha$ and $I L-1 \beta$ and similar levels of expression of $I L-1 R A$, compared to the basal medium.
$T N F-\alpha, P T G E S, T G F-\beta 1$, and $H G F$ experienced differential up- and downregulation in normal, type I diabetic, and type II diabetic ADSCs as well. For example, type I diabetic ADSCs significantly upregulated $T N F-\alpha$ expression at 5 and $10 \mathrm{mg} / \mathrm{mL}$ (Fig. 6d), type II diabetic ADSCs downregulated PTGES expression at $10 \mathrm{mg} / \mathrm{mL}$ (Fig. 6e), type II diabetic ADSCs downregulated TGF- $\beta 1$ expression at 5 and $10 \mathrm{mg} / \mathrm{mL}$ (Fig. 6f), and type I and type II diabetic ADSCs also significantly downregulated $H G F$ expression in a dose-dependent manner following dHACM treatment (Fig. 6g). For comparison, in complete medium that represented the standard culture conditions for these cells, diabetic ADSCs consistently had a higher expression of $T G F-\beta 1$, a lower expression of PTGES and $H G F$, and similar levels of expression of $T N F-\alpha$, compared to the basal medium. These data suggest that ADSCs isolated from normal and diabetic donors respond to treatment with dHACM by altering their gene expression of various paracrine signaling factors and cytokines.

\section{DISCUSSION}

This study evaluated dHACM for the induction of cellular responses from ADSCs derived from diabetic donors, which are clinically relevant to 
a IL-1 $\alpha$

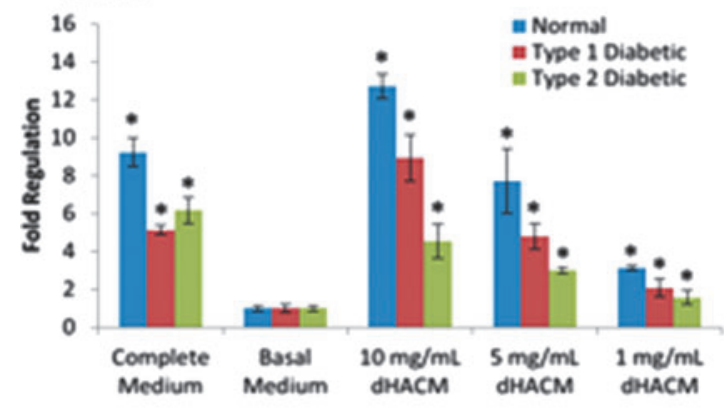

C IL-1RA

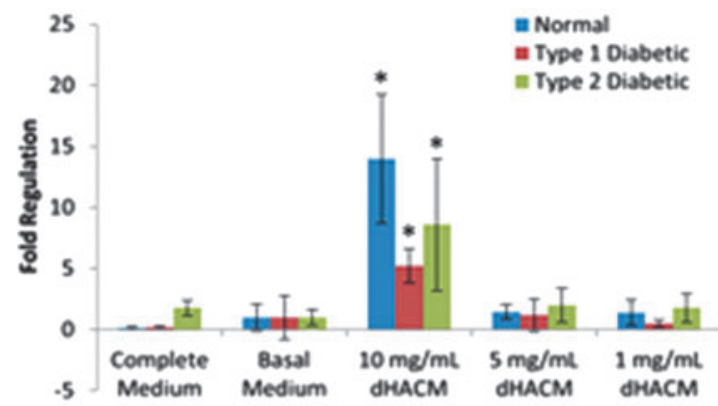

e PTGES

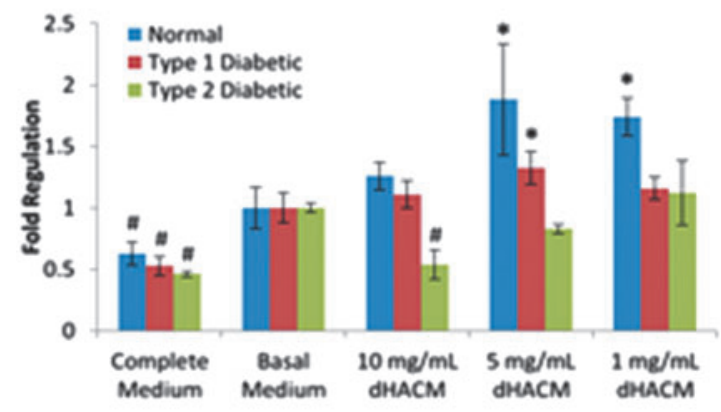

b IL-1 $\beta$

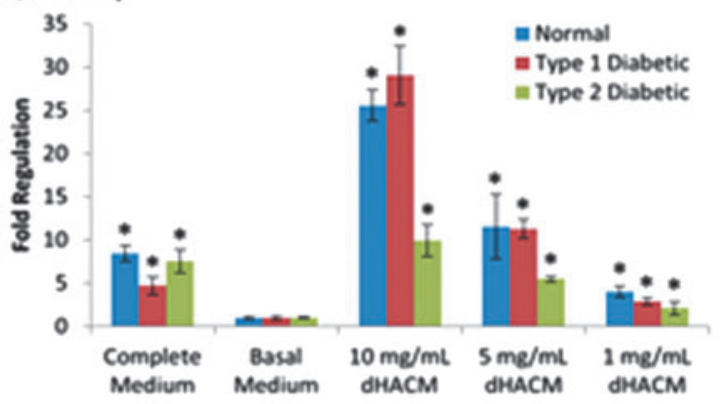

d TNF

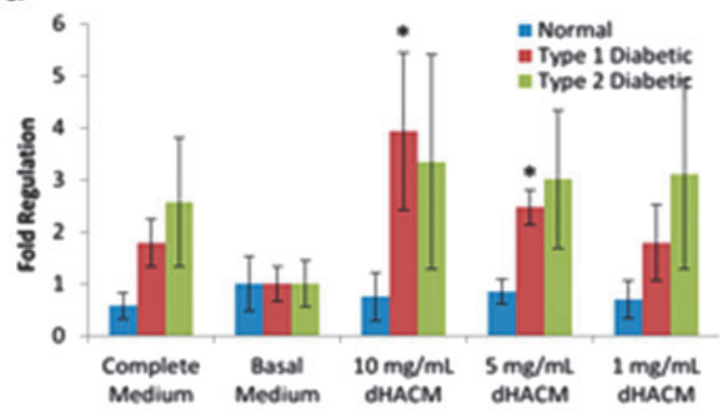

f TGF- $\beta 1$

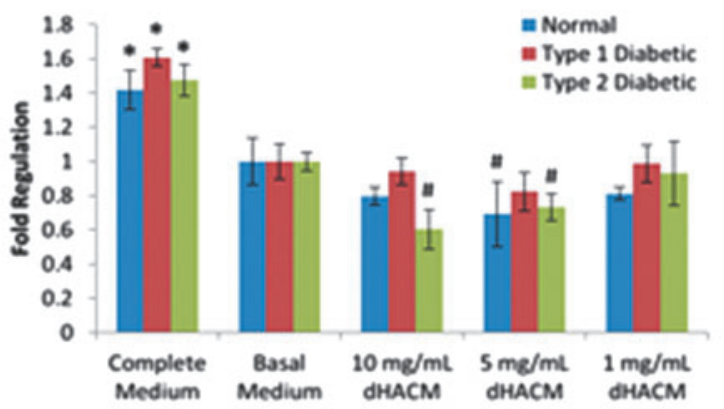

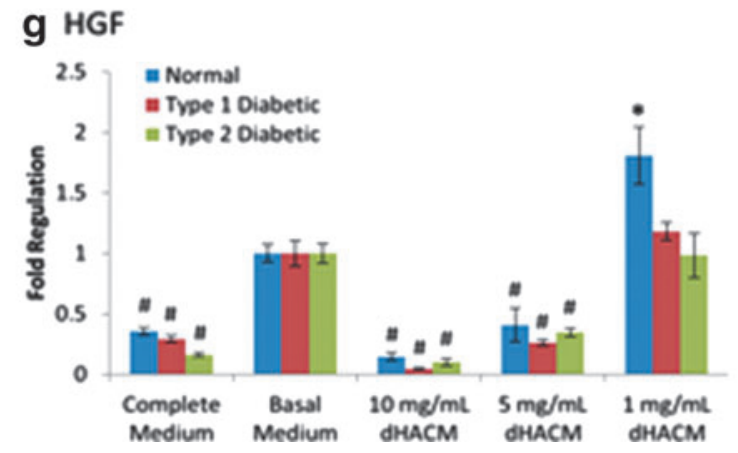

Figure 6. Gene expression of dHACM-treated normal, type I diabetic, and type II diabetic ADSCs. Fold regulation of (a) IL-1 $\alpha$, (b) IL-1 $\beta$, (c) IL-1RA, (d) TNF- $\alpha$, (e) PTGES, (f) TGF- $\beta 1$, and (g) HGF is expressed relative to ADSCs cultured in basal medium (fold regulation=1). *Significant upregulation from basal medium $(p \leq 0.05)$. " Significant downregulation from basal medium ( $p \leq 0.05)$. HGF, hepatocyte growth factor; IL, interleukins; PTGES, prostaglandin E synthase; TGF- $\beta$, transforming growth factor-beta; TNF- $\alpha$, tumor necrosis factor- $\alpha$.

many patients receiving dHACM treatment of chronic wounds, including DFUs and venous leg ulcers (VLUs). Successful clinical results demonstrate the healing capacity of dHACM. ${ }^{21,24-26} \mathrm{~A}$ study comprised of patients with type I or type II diabetes presenting with chronic DFUs were treated with weekly or biweekly applications of dHACM. Complete wound healing was achieved in 37 of the 40 patients at the 12 -week study mark. ${ }^{25}$ In addition, a prospective randomized 
controlled trial for the treatment of DFUs showed that $77 \%$ and $92 \%$ of chronic wounds at 4 and 6 weeks, respectively, healed with biweekly dHACM treatment. Of these healed patients, 94\% remained fully healed after 9-12 months of follow-up ${ }^{24}$ compared to the standard of care that only healed 0 and $8 \%$, respectively. ${ }^{21}$ The goal of this study was to elucidate an important aspect of the mechanism of action of dHACM for accelerating chronic wound healing, as observed clinically in diabetic patients, by demonstrating the impact of dHACM on diabetic stem cell proliferation, migration, and gene expression.

These studies confirmed that type I and II diabetic ADSCs had impaired proliferation and migratory (type I only) potential, and altered growth factor and cytokine production in comparison to normal ADSCs in vitro. The growth factors and cytokines that differed in expression among ADSCs tested were mainly immunomodulatory factors and these were expressed at highest levels in type II diabetic ADSCs.

The results of this study suggest that dHACM extracts are capable of stimulating cellular proliferation and migration responses of ADSCs from type I and type II diabetic donors. The impairment imparted by diabetes on ADSCs is partially overcome through the exposure to the eluted soluble growth factors and cytokines from dHACM in vitro. Due to the complexity of this unique tissue, which contains a multitude of growth factors and cytokines, a causative effect cannot be assigned to any single factor or regulatory protein; however, it is more than likely a synergistic effect of combined factors. The observed increases in diabetic ADSC proliferation and migration in response to the soluble extracts of dHACM were accompanied by modulation of the secretion profile of immunomodulatory proteins from each of the cell lines tested. The results showed an increase in the immunomodulatory factors IL-6, IL-8, MCP-1, MIP-1 $\beta$, and RANTES, secreted by type I and type II diabetic ADSCs with treatment, which are recognized as being responsible for the inflammatory regulation functionality of ADSCs in wound healing. ${ }^{14}$ For example, IL-6-deficient mice present delayed healing and reepithelialization, suggesting that IL-6 is pleiotropic, playing an essential role in leukocyte infiltration, angiogenesis, and collagen accumulation. ${ }^{27}$ Many of these factors work as chemoattractants for other cells, which have a positive downstream impact on the healing process. MCP-1 recruits mast cells that produce high levels of IL-4 and, in turn, stimulates the proliferation of fibroblasts and aids in transitioning out of the inflammatory phase by downregulating the expression of proinflammatory chemokines. ${ }^{28}$ Type II diabetic ADSCs also increased expression of VEGF with treatment, suggesting that these ADSCs have the potential to respond to dHACM by contributing angiogenic factors that stimulate tissue vascularization. Thus, initial upregulation of certain immunomodulatory factors through treatment with dHACM may be necessary for resetting the cascade of normal healing in a chronic wound.

Gene expression analysis of type I and type II diabetic ADSCs in complete medium suggested that diabetic cells possessed an altered gene expression profile of a number of genes related to ECM, adhesion, cytokines, and diabetes, compared to ADSCs from healthy donors. Most notably, however, diabetic ADSCs had greater expression of a number of immunomodulatory factors. For example, IL- $1 \alpha$, IL-1 $\beta$, and TNF- $\alpha$, along with IL-1RA, prostaglandin E2, TGF- $\beta 1$, and HGF are believed to play important roles in the immunoregulation of wound environments by MSCs and ADSCs, including modulation of B-cell and T-cell responses. ${ }^{29-31} \mathrm{It}$ was also observed that gene expression of $I L-1 \alpha$ and $I L-1 \beta$ was upregulated by both normal and diabetic ADSCs following 5 days of treatment with dHACM extracts. However, expression of $I L-1 R A$, the receptor antagonist of IL-1 signaling was also upregulated in all cell types with $10 \mathrm{mg} / \mathrm{mL}$ dHACM treatment. This suggests that dHACM mediates a balanced response in ADSCs that impacts expression of both proinflammatory and antiinflammatory immunomodulatory factors which, in turn, may contribute to providing a balance of stimuli altering the chronically inflamed status of a wound to one of an acute healing trajectory.

The commercial availability of diabetic ADSC donors was limited resulting in the inclusion of a single donor for each diabetic etiology; therefore, the data collected were compared to control groups within the individual donor to measure responsiveness to dHACM. In addition, it is possible that these observations are unique to in vitro conditions. Additional studies would be required to make the correlation to in vivo scenarios; however, previous rodent studies have demonstrated the in vivo angiogenic potential of dHACM following implantation in ischemic tissues, ${ }^{18}$ and positive clinical outcomes for healing DFU and VLUs suggest that dHACM modulates the cellular environment in a diabetic and/or ischemic wound bed. ${ }^{21,24-26}$

\section{INNOVATION}

dHACM allografts have proven effective for treatment of chronic wounds, including DFUs. 
dHACM grafts have been shown to recruit adult stem cells and alter their activity in vivo and in vitro; however, it is currently not well understood how the effects of diabetes may impair the activity or responsiveness of these reparative stem cells. The studies presented here demonstrated that diabetic ADSCs proliferated, migrated, and altered expression of immunomodulatory cytokines in response to dHACM treatment. Therefore, this is the first reported case demonstrating that diabetic ADSCs respond to novel amniotic membrane therapies, such as dHACM treatment.

\section{ACKNOWLEDGMENTS AND FUNDING SOURCES}

This work was funded by MiMedx Group, Inc. M.M., K.C., J.J.L., L.G., C.S.Y., and T.J.K. are employees of MiMedx.

\section{AUTHOR DISCLOSURE AND GHOSTWRITING}

M.M., K.C., J.J.L., L.G., C.S.Y., and T.J.K. are employees of MiMedx. The content of this article was expressly written by M.M., K.C., J.J.L., L.G., C.S.Y., and T.J.K. No ghostwriters were used to write this article.

\section{ABOUT THE AUTHORS}

Michelle Massee is the R\&D department Biomedical Project Manager. She received her bachelor's degree in Biomedical Engineering from the Georgia Institute of Technology. Kathryn Chinn is a Re-

\section{KEY FINDINGS}

- dHACM grafts can biologically stimulate type I and II diabetic ADSCs.

- $\mathrm{dHACM}$ grafts promote proliferation and migration of type I and II diabetic ADSCs.

- dHACM modulates type I and II diabetic environments by regulating endogenous production of a variety of soluble signals by stem cells, including regulators of inflammation, mitogenesis, and wound healing.

search Technician within the R\&D department of MiMedx. She received her bachelor's degree in Biological Engineering from the University of Florida. Jeremy J. Lim, PhD, is the Senior Biomedical Engineer within the R\&D department of MiMedx. He received his $\mathrm{PhD}$ in Biomedical Engineering from the Georgia Institute of Technology. Lisa Godwin is a Research Technician within the R\&D department of MiMedx. She received her associate degree in Medical Laboratory Technology from Georgia Baptist. Conan Young, PhD, is the Director of Research within the R\&D department of MiMedx. He received his $\mathrm{PhD}$ in Microbiology from the University of British Columbia. Thomas J. Koob, PhD, is the Chief Scientific Officer of MiMedx. He is the senior executive responsible for the overall research direction and oversight of the company's product and tissue offerings. Dr. Koob received his PhD in Biochemistry from Washington University School of Medicine in St. Louis, Missouri. Dr. Koob has published over 145 biomedical and biological articles and 12 book chapters, and he is listed as an inventor on over 80 issued and pending patents.

\section{REFERENCES}

1. Diabetes. World Health Organization 2015; Fact Sheet No 312.

2. Evans KK, Attinger CE, Al-Attar A, et al. The importance of limb preservation in the diabetic population. J Diabetes Complications 2011;25: 227-231.

3. Tsourdi E, Barthel A, Rietzsch H, Reichel A, Bornstein SR. Current aspects in the pathophysiology and treatment of chronic wounds in diabetes mellitus. Biomed Res Int 2013;2013:385641

4. LoGerfo FW, Coffman JD. Current concepts. Vascular and microvascular disease of the foot in diabetes. Implications for foot care. N Engl J Med 1984;311:1615-1619.

5. Lauer G, Sollberg S, Cole M, et al. Expression and proteolysis of vascular endothelial growth factor is increased in chronic wounds. J Invest Dermatol 2000;115:12-18.
6. Jude EB, Blakytny R, Bulmer J, Boulton AJ, Ferguson MW. Transforming growth factor-beta 1, 2 , 3 and receptor type I and II in diabetic foot ulcers. Diabet Med 2002;19:440-447.

7. Wu Y, Wang J, Scott PG, Tredget EE. Bone marrow-derived stem cells in wound healing: a review. Wound Repair Regen 2007;15 Suppl 1:S18-S26.

8. Izadpanah R, Trygg C, Patel B, et al. Biologic properties of mesenchymal stem cells derived from bone marrow and adipose tissue. J Cell Biochem 2006;99:1285-1297.

9. Zuk P. Adipose-derived stem cells in tissue regeneration: a review. ISRN Stem Cells 2013;2013:35.

10. Gimble JM, Katz AJ, Bunnell BA. Adipose-derived stem cells for regenerative medicine. Circ Res 2007; 100:1249-1260.
11. Kim EK, Li G, Lee TJ, Hong JP. The effect of human adipose-

derived stem cells on healing of ischemic wounds in a diabetic nude mouse model. Plast Reconstr Surg 2011;128:387-394.

12. Nambu M, Kishimoto $S$, Nakamura $S$, et al. Accelerated wound healing in healing-impaired $\mathrm{db} /$ $\mathrm{db}$ mice by autologous adipose tissue-derived stromal cells combined with atelocollagen matrix. Ann Plast Surg 2009;62:317-321.

13. Salgado AJ, Reis RL, Sousa NJ, Gimble JM. Adipose tissue derived stem cells secretome: soluble factors and their roles in regenerative medicine. Curr Stem Cell Res Ther 2010;5:103-110.

14. Nie C, Yang D, Xu J, Si Z, Jin X, Zhang J. Locally administered adipose-derived stem cells accelerate wound healing through differentiation and vasculogenesis. Cell Transplant 2011;20:205-216. 
15. Cramer C, Freisinger E, Jones RK, et al. Persistent high glucose concentrations alter the regenerative potential of mesenchymal stem cells. Stem Cells Dev 2010;19:1875-1884.

16. Cianfarani F, Toietta G, Di Rocco G, Cesareo E, Zambruno G, Odorisio T. Diabetes impairs adipose tissue-derived stem cell function and efficiency in promoting wound healing. Wound Repair Regen 2013:21:545-553.

17. Massee M, Chinn K, Lei J, Lim JJ, Young CS, Koob TJ. Dehydrated human amnion/chorion membrane regulates stem cell activity. J Biomed Mater Res B Appl Biomater 2015; D0I: 10.1002/jbm.b.33478.

18. Koob TJ, Lim JJ, Massee M, Zabek N, Denoziere G. Properties of dehydrated human amnion/chorion composite grafts: implications for wound repair and soft tissue regeneration. J Biomed Mater Res B Appl Biomater 2014;102:1353-1362.

19. Koob TJ, Lim JJ, Massee M, et al. Angiogenic properties of dehydrated human amnion/chorion allografts: therapeutic potential for soft tissue repair and regeneration. Vasc Cell 2014;6:10.

20. Koob TJ, Lim JJ, Zabek N, Massee M. Cytokines in single layer amnion allografts compared to multilayer amnion/chorion allografts for wound healing. J Biomed Mater Res B Appl Biomater 2015;103: 1133-1140.

21. Zelen CM, Serena TE, Denoziere G, Fetterolf DE. A prospective randomised comparative parallel study of amniotic membrane wound graft in the management of diabetic foot ulcers. Int Wound $\mathrm{J}$ 2013;10:502-507.

22. Daniel J, Tofe R, Spencer R, Russo J, inventors; MiMedx Group, Inc (Kennesaw, GA), assignee. Placental Tissue Grafts. 2013; US Patent 8,409,626.

23. Livak KJ, Schmittgen TD. Analysis of relative gene expression data using real-time quantitative PCR and the 2(-Delta Delta $C(T))$ method. Methods 2001;25:402-408.

24. Zelen CM, Serena TE, Fetterolf DE. Dehydrated human amnion/chorion membrane allografts in patients with chronic diabetic foot ulcers: a longterm follow-up study. Wound Medicine 2014;4:1-4.

25. Zelen CM, Serena TE, Snyder RJ. A prospective, randomised comparative study of weekly versus biweekly application of dehydrated human amnion/ chorion membrane allograft in the management of diabetic foot ulcers. Int Wound J 2014;11:122-128.

26. Serena TE, Carter MJ, Le LT, Sabo MJ, DiMarco DT, EpiFix VLUSG. A multicenter, randomized, controlled clinical trial evaluating the use of dehydrated human amnion/chorion membrane allografts and multilayer compression therapy vs. multilayer compression therapy alone in the treatment of venous leg ulcers. Wound Repair Regen 2014;22:688-693.

27. Lin ZQ, Kondo T, Ishida Y, Takayasu T, Mukaida N. Essential involvement of IL-6 in the skin woundhealing process as evidenced by delayed wound healing in IL-6-deficient mice. J Leukoc Biol 2003;73:713-721.

28. Leonard EJ, Skeel A, Yoshimura T, Rankin J. Secretion of monocyte chemoattractant protein-1 (MCP-1) by human mononuclear phagocytes. Adv Exp Med Biol 1993;351:55-64.

29. Chen Y, Shao JZ, Xiang LX, Dong XJ, Zhang GR. Mesenchymal stem cells: a promising candidate in regenerative medicine. Int $\mathrm{J}$ Biochem Cell Biol 2008;40:815-820.

30. Ortiz LA, Dutreil M, Fattman C, et al. Interleukin 1 receptor antagonist mediates the antiinflammatory and antifibrotic effect of mesenchymal stem cells during lung injury. Proc Natl Acad Sci U S A 2007;104:11002-11007.

31. Ryan JM, Barry FP, Murphy JM, Mahon BP. Mesenchymal stem cells avoid allogeneic rejection. J Inflamm (Lond) 2005;2:8.

\section{Abbreviations and Acronyms}

$\mathrm{AATB}=$ American Association of Tissue Banks

ADSCs $=$ adipose-derived stem cells

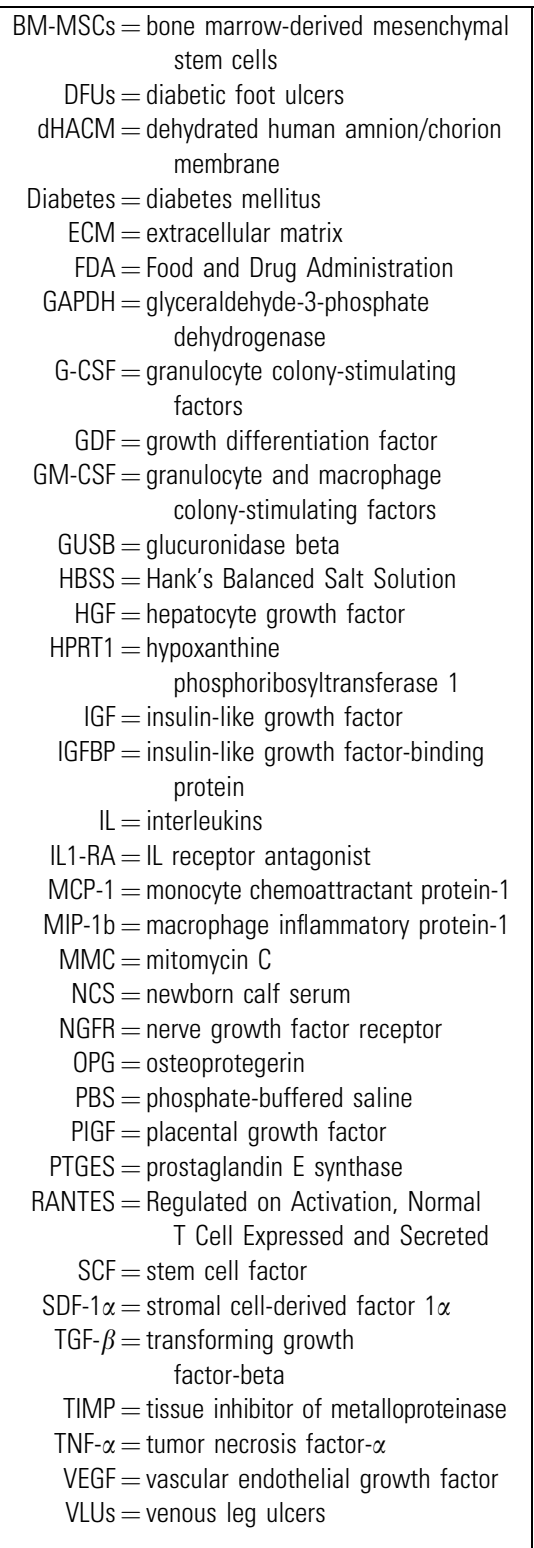

